\section{Atenção à saúde da criança: uma análise do grau de implantação e da satisfação de profissionais e usuários em dois municípios do estado de Pernambuco, Brasil}

\author{
Child health care: implementation degree \\ analysis and satisfaction of health \\ professionals and users in two \\ municipalities at Pernambuco state, Brazil
}

Isabella Samico 1

Zulmira Maria de Araújo Hartz 2

Eronildo Felisberto 3

Eduardo Freese de Carvalho 4

${ }^{1}$ Instituto Materno Infantil Professor Fernando Figueira (IMIP). Rua dos Coelhos, 300. Recife, PE, Brasil. CEP: 50.070-550

2 Professora-pesquisadora convidada do Departamento de

Medicina Preventiva e Social (DMPS) e Groupe de Recherche Interdisciplinaire en Santé (GRIS) da Universidade de Montreal, Canadá.

3Ministério da Saúde. Secretaria de Atenção à Saúde.

Departamento de Atenção Básica. Coordenação de Acompanhamento e Avaliação. Brasília, DF, Brasil.

4 Núcleo de Estudos em Saúde Coletiva. Centro de Pesquisas

Aggeu Magalhães. Fundação Oswaldo Cruz. Recife, PE, Brasil.

\section{Resumo}

Objetivos: avaliar a implantação da atenção à saúde da criança em dois municípios do estado de Pernambuco, Brasil.

Métodos: pesquisa avaliativa, tipo análise de implantação. Consideradas três unidades de análise: unidade do Programa Saúde da Familia (PSF) com profissionais treinados na Estratégia Atenção Integrada às Doenças Prevalentes da Infância (AIDPI), unidade sem esse treinamento e unidade tradicional. Para obtenção do grau de implantação foi realizada observação do manejo de casos e estru tura. A percepção dos profissionais e usuários foi apreendida por meio de entrevista e discussão em grupos focais.

Resultados: grau de implantação satisfatório para unidades do PSF, insatisfatório para unidade tradicional no Município 1 e insatisfatório para unidades do Municipio 2. Os profissionais consideraram bons a resolubilidade dos serviços e o relacionamento inter pessoal, mas dificuldades no sistema de referência e falta de integração das equipes das unidades tradi cionais. Os usuários relataram boa resolubilidade, mas dificuldades no acesso e nas relações interpes soais.

Conclusões: a variação no grau de implantação apresentou como determinantes deficiências no manejo de casos e potencialidade da atenção prestada pelo enfermeiro. Não foi possivel definir influência da AIDPI. Há melhor satisfação profis sional e dos usuários para unidades do PSF, porém problemas no acesso e integralidade da atenção.

Palavras-chave Avaliação de programas, Avaliação de serviços, Saúde infantil (Saúde pública), Satisfação dos consumidores 


\section{Introdução}

A atenção à saúde da criança representa um campo prioritário dentro dos cuidados à saúde das populações. Para que essa se desenvolva de forma mais efetiva e eficiente, além do conhecimento sobre as características relacionadas à morbi-mortalidade, tais como aspectos biológicos, demográficos e socioeconômicos, é importante salientar o papel que desempenham os serviços e o sistema de saúde. Problemas no cumprimento de normas técnicas por parte dos profissionais, não realização de trabalhos educativos, falta de equipamentos e outros insumos, deficiências na notificação de dados e dificuldades no processo de trabalho dos profissionais são pontos que persistem e que são, de forma evidente, impeditivos para uma adequada atenção à saúde. ${ }^{1}$

Nesse contexto, os cuidados primários em saúde apresentam grande relevância como uma possibilidade para o enfrentamento dos problemas. Casanova e Starfield 2 salientam que um sistema de atenção primária adequado é capaz de reduzir a ocorrência de situações indicativas de cuidados insatisfatórios como ausência de ações de prevenção de doenças e promoção da saúde, longos intervalos para visitas de seguimento, dificuldades de acesso a serviços de referência, além de um excesso de hospitalizações por problemas passíveis de resolução mediante uma atenção primária satisfatória.

A implantação do Programa Saúde da Família (PSF) e a Estratégia de Atenção Integrada às Doenças Prevalentes da Infância (AIDPI), incorporadas à política de saúde do Brasil, vêm possibilitando um maior acesso aos cuidados básicos de saúde. Essas iniciativas propõem tornar mais resolutivo o âmbito da atenção primária. ${ }^{3-5}$

Para se compreender de que forma e em que medida as ações, programas e políticas são implantadas e conhecer os efeitos dessas intervenções, há que se desenvolver processos avaliativos pertinentes e oportunos. A avaliação deve, portanto, ser compreendida como uma ferramenta relacionada à tomada de decisão. Nesse sentido, Bodstein ${ }^{6}$ salienta a importância dos processos avaliativos, em especial no âmbito local, em face da política de descentralização do Sistema Único de Saúde. Diante de contextos diversos, reforça a utilidade de análises qualitativas, comparativas e de estudos de caso para o planejamento e a gestão, considerando o fortalecimento das ações básicas e o desenvolvimento da atenção primária, efetivos de fato para uma melhor oferta de saúde.

Este estudo teve como objetivo avaliar a implantação da atenção à saúde da criança na rede básica de saúde em dois municípios do estado de Pernambuco.

\section{Métodos}

Pesquisa avaliativa do tipo análise da implantação tendo como estratégia o estudo de casos múltiplos com níveis de análise imbricados.

O estudo foi realizado em dois municípios localizados na Região Agreste do estado de Pernambuco durante o período de abril a novembro de 2002. O município 1 foi selecionado por ter sido um dos primeiros a implantar o PSF no estado de Pernambuco, a partir de 1996, e a Estratégia AIDPI a partir de 1998. Em decorrência do modelo metodológico adotado, houve necessidade da inclusão de um segundo município com características sóciodemográficas semelhantes no sentido da reprodutibilidade dos resultados.

Foram considerados três serviços de saúde em cada município selecionado: duas unidades do PSF, um das quais dispunha de profissionais capacitados na estratégia AIDPI (PSF com AIDPI), uma com profissionais não capacitados nesta estratégia (PSF sem AIDPI) e uma pertencente ao modelo tradicional de atenção à saúde (unidade tradicional). Para o estado de Pernambuco, o treinamento na estratégia AIDPI foi priorizado para os profissionais de nível superior do Programa Saúde da Família. Todas as unidades estavam localizadas na área urbana dos municípios.

Cada caso apresenta cada um dos serviços (unidades de análise) considerados em três níveis de análise: um primeiro, apoiado na própria unidade de saúde mediante caracterização das dimensões estruturais, do processo de trabalho e da percepção dos profissionais e usuários; um segundo, apoiado na comunidade, procurando apreender sua inter-relação com os serviços de saúde e as percepções sobre a atenção à saúde, sob a ótica da atenção primária; e um terceiro nível, tomando para análise o sistema de referência e contra-referência com os serviços de apoio secundário e/ou terciário. Este artigo abordará o primeiro nível de análise.

A população do estudo foi composta por crianças menores de cinco anos, pais ou responsáveis e profissionais de saúde das unidades selecionadas.

O grau de implantação da atenção à saúde da criança foi definido a partir da análise da estrutura dos serviços de saúde e do processo de trabalho, e este a partir da observação do manejo de casos de crianças menores de cinco anos de vida realizado pelos profissionais responsáveis pela atenção à saúde da criança e da percepção dos usuários após o atendimento. Foi construído um sistema de escores adaptado a partir do método proposto por Felisberto et al. ${ }^{7}$ Para o escore final, foi imputado peso 6 ao manejo de casos e peso 
4 à estrutura. Consideraram-se como pontos de corte para classificação do grau de implantação: Crítico $=0$ a 4,9; Insatisfatório $=5,0$ a 7,9; Satisfatório $=8,0$ a 10,0 . As variáveis foram definidas a partir de adaptação do Manual de Acompanhamento e Avaliação da Estratégia AIDPI. ${ }^{8}$ Foram consideradas as seguintes variáveis (com os respectivos pesos). Para estrutura: "medicamentos" (2,5), "vacinas" $(2,5)$, "equipamentos e outros insumos" $(2,5)$ e "organização de serviços" $(2,5)$; para processo: "avaliação clínica" $(2,5)$ nessa considerando a verificação de afecções prevalentes, outros problemas e estado nutricional, "tratamento" $(2,5)$ nesse incluindo medicamentoso e referência, "ações de promoção da saúde e prevenção de doenças" $(2,0)$ quanto a orientações sobre alimentação, imunização, crescimento e desenvolvimento, retorno ao serviços e notificação compulsória de doenças, "habilidades de comunicação" $(1,0)$ quanto a orientações sobre tratamento e perguntas de verificação e "percepção dos usuários após o atendimento" $(2,0)$ considerando a compreensão satisfatória sobre o problema de saúde, o tratamento e as ações de promoção da saúde e prevenção de doenças quanto à alimentação, imunização, crescimento e desenvolvimento e retorno ao serviço.

Para a análise da percepção dos profissionais de saúde foram definidas as seguintes dimensões: suporte técnico, resolubilidade do serviço e relações pessoais e interpessoais. Os dados foram coletados por meio de entrevista semi-estruturada com os profissionais médicos e de enfermagem. Para percepção dos usuários das unidades de saúde, as dimensões consideradas foram: acesso e resolubilidade do serviço, modelos de atenção à saúde e satisfação com os serviços prestados. A coleta dos dados foi realizada por meio de discussão em grupos focais com pais ou responsáveis por crianças menores de cinco anos.

Para a análise estatística dos dados, foi utilizado o software Epi-info. A análise de conteúdo foi empregada para os dados de natureza qualitativa. ${ }^{9}$

\section{Resultados}

\section{Grau de implantação da atenção}

\section{à saúde da criança menor de cinco anos}

Para o Município 1, o grau de implantação apresentou-se satisfatório para as unidades pertencentes ao PSF e insatisfatório para a unidade tradicional. Para o Município 2, a implantação da atenção à saúde da criança mostrou-se insatisfatória para todas as unidades.
$\mathrm{Na}$ Tabela 1 encontram-se os resultados dos escores para o manejo de casos e estrutura. Em ambos os casos pode ser observado que a maior parte dos escores referentes à unidade tradicional apresenta uma pior situação em relação às demais unidades.

Em relação ao manejo de casos para o Município 1 observam-se melhores escores para a unidade do PSF sem treinamento em AIDPI, em que pese o fato de que o profissional médico não realizava atenção à criança de forma regular, porém o profissional de enfermagem o fazia e utilizava a estratégia AIDPI. Embora essa unidade tenha sido definida para o estudo com o critério do não treinamento nessa estratégia, foi verificado que todos os profissionais de enfermagem das unidades do PSF do município receberam esse treinamento. Na unidade PSF com AIDPI o profissional médico treinado na estratégiaAIDPI referiu não a utilizar devido ao tempo escasso para a quantidade de atendimentos, pois a sistematização da consulta segundo tal estratégia AIDPI requereria um período de tempo mais longo. A unidade tradicional, um centro de saúde, contava com seis médicos pediatras e um enfermeiro, este treinado e utilizando na prática a estratégia AIDPI.

Para o Município 2 os escores relativos ao manejo de casos apresentam-se mais baixos para a unidade PSF com AIDPI. Nessa, o profissional médico, embora treinado na estratégia, não a utilizava na prática. Em ambas as unidades do PSF, os profissionais de enfermagem não realizavam atenção à criança. A unidade tradicional, uma unidade mista, contava com três médicos pediatras para a atenção à saúde da criança.

Deve-se observar que, para os dois municípios, os escores relacionados aos procedimentos terapêuticos apresentam-se satisfatórios. Entretanto, de forma geral, os escores relacionados às ações de prevenção e promoção encontram-se abaixo do total atribuído para as variáveis de ambas as dimensões (observação do manejo de casos e percepção dos usuários após o atendimento).

Ao se observarem os escores obtidos para estrutura, ressalte-se que para ambos os casos esses se apresentam satisfatórios, à exceção da unidade tradicional do Município 1, porém próximo à classificação "satisfatório". Observam-se escores menores relacionados à organização dos serviços para as unidades pertencentes ao modelo tradicional.

$\mathrm{Na}$ Tabela 2 pode ser observado o escore final com o grau de implantação para cada uma das unidades avaliadas. Observa-se que, embora com implantação insatisfatória, a unidade do PSF sem AIDPI do Município 2 apresenta um escore final próximo à faixa limite inferior considerada, para a classificação, como "satisfatória". 
Escores para o manejo de casos de crianças menores de cinco anos e para estrutura nas unidades de saúde avaliadas nos Municípios 1 e 2 , em 2002.

\begin{tabular}{|c|c|c|c|c|c|c|}
\hline \multirow[b]{2}{*}{ Dimensão / Variável (Peso total)* } & \multicolumn{3}{|c|}{ Município 1} & \multicolumn{3}{|c|}{ Município 2} \\
\hline & $\begin{array}{l}\text { PSF com } \\
\text { AIDPI }\end{array}$ & $\begin{array}{l}\text { PSF sem } \\
\text { AIDPI }\end{array}$ & $\begin{array}{l}\text { Unidade } \\
\text { tradicional }\end{array}$ & $\begin{array}{l}\text { PSF com } \\
\text { AIDPI }\end{array}$ & $\begin{array}{l}\text { PSF sem } \\
\text { AIDPI }\end{array}$ & $\begin{array}{l}\text { Unidade } \\
\text { tradicional }\end{array}$ \\
\hline \multicolumn{7}{|l|}{ Observação do manejo de casos: } \\
\hline \multicolumn{7}{|l|}{ Avaliação clínica $(2,5)$ : } \\
\hline Afecções prevalentes $(1,0)$ & 0,61 & 0,88 & 0,42 & 0,40 & 0,53 & 0,31 \\
\hline Outros problemas $(0,75)$ & 0,60 & 0,75 & 0,48 & 0,75 & 00 & 0,15 \\
\hline Estado nutricional $(0,75)$ & 0,52 & 0,42 & 0,36 & 00 & 0,60 & 0,07 \\
\hline \multicolumn{7}{|l|}{ Tratamento $(2,5)$ : } \\
\hline Medicamentoso $(1,25)$ & 1,25 & 1,25 & 0,83 & 0,83 & 1,00 & 1,25 \\
\hline Referência $(1,25)$ & 1,25 & 1,25 & 1,25 & 1,25 & 1,25 & 1,25 \\
\hline \multicolumn{7}{|l|}{$\begin{array}{l}\text { Ações de promoção da saúde e } \\
\text { prevenção de doenças }(2,0) \text { : }\end{array}$} \\
\hline Orientações sobre alimentação $(0,5)$ & 0,40 & 0,42 & 0,20 & 00 & 0,20 & 0,05 \\
\hline Imunização $(0,5)$ & 0,40 & 0,42 & 0,14 & 00 & 0,40 & 0,20 \\
\hline Crescimento e desenvolvimento $(0,5)$ & 0,35 & 0,28 & 0,10 & 00 & 0,40 & 0,05 \\
\hline Retomo ao serviço $(0,25)$ & 0,25 & 0,14 & 0,16 & 0,15 & 0,15 & 0,07 \\
\hline Notificação compulsória de doenças $(0,25)$ & 0,25 & 0,25 & 0,25 & 0,25 & 0,25 & 0,25 \\
\hline \multicolumn{7}{|l|}{ Habilidades de comunicação $(1,0)$ : } \\
\hline Orientações sobre o tratamento $(0,75)$ & 0,75 & 0,75 & 0,72 & 0,75 & 0,75 & 0,75 \\
\hline Perguntas de verificação $(0,25)$ & 00 & 00 & 00 & 0,08 & 00 & 00 \\
\hline \multicolumn{7}{|l|}{ Percepção dos usuários após o atendimento: } \\
\hline \multicolumn{7}{|l|}{ Compreensão satisfatória sobre $(1,0)$ : } \\
\hline Problema de saúde $(0,5)$ & 0,50 & 0,33 & 0,32 & 0,30 & 0,50 & 0,50 \\
\hline Tratamento $(0,5)$ & 0,50 & 0,50 & 0,22 & 0,40 & 0,20 & 0,16 \\
\hline \multicolumn{7}{|l|}{$\begin{array}{l}\text { Ações de promoção da saúde e } \\
\text { prevenção de doenças }(1,0) \text { : }\end{array}$} \\
\hline Alimentação $(0,25)$ & 0,10 & 0,14 & 0,09 & 00 & 0,05 & 0,06 \\
\hline Imunização $(0,25)$ & 0,15 & 0,25 & 0,06 & 00 & 0,10 & 0,06 \\
\hline Crescimento e desenvolvimento $(0,25)$ & 0,07 & 0,10 & 0,05 & 00 & 0,05 & 00 \\
\hline Retomo ao serviço $(0,25)$ & 0,20 & 0,25 & 0,16 & 00 & 0,20 & 0,03 \\
\hline Total - Manejo de casos & 8,15 & 8,38 & 5,81 & 5,16 & 6,63 & 5,21 \\
\hline \multicolumn{7}{|l|}{ Estrutura } \\
\hline Medicamentos $(2,5)$ & 2,50 & 2,00 & 1,75 & 2,25 & 2,25 & 2,50 \\
\hline Vacinas $(2,5)$ & 2,50 & 2,50 & 2,50 & 2,50 & 2,50 & 2,50 \\
\hline Equipamentos/outros insumos $(2,5)$ & 2,34 & 1,97 & 1,80 & 2,34 & 2,14 & 2,17 \\
\hline Organização de serviços $(2,5)$ & 2.50 & 1,75 & 1,68 & 1,75 & 2,00 & 1,00 \\
\hline Total estrutura & 9,84 & 8,22 & 7,73 & 8,84 & 8,89 & 8,17 \\
\hline
\end{tabular}

* Crítico = 0 a 4,9; Insatisfatório = 5,0 a 7,9; Satisfatório = 8,0 a 10,0. 
Escore final e grau de implantação para as unidades de saúde avaliadas nos Municípios 1 e 2, em 2002.

\begin{tabular}{|c|c|c|c|c|c|c|}
\hline \multirow[b]{2}{*}{ Dimensão* } & \multicolumn{3}{|c|}{ Município 1} & \multicolumn{3}{|c|}{ Município 2} \\
\hline & $\begin{array}{l}\text { PSF com } \\
\text { AIDPI }\end{array}$ & $\begin{array}{l}\text { PSF sem } \\
\text { AIDPI }\end{array}$ & $\begin{array}{c}\text { Unidade } \\
\text { tradicional }\end{array}$ & $\begin{array}{l}\text { PSF com } \\
\text { AIDPI }\end{array}$ & $\begin{array}{l}\text { PSF sem } \\
\text { AIDPI }\end{array}$ & $\begin{array}{c}\text { Unidade } \\
\text { tradicional }\end{array}$ \\
\hline Manejo de casos & 8,15 & 8,38 & 5,81 & 5,16 & 6,63 & 5,21 \\
\hline Estrutura & 9,84 & 8,22 & 7,73 & 8,84 & 8,89 & 8,17 \\
\hline Escore final ** & 8,82 & 8,31 & 6,57 & 6,63 & 7,53 & 6,39 \\
\hline $\begin{array}{l}\text { Grau de implanta } \\
\text { da criança }\end{array}$ & Satisfatório & Satisfatório & Insatisfatório & Insatisfatório & Insatisfatório & Insatisfatório \\
\hline
\end{tabular}

* Crítico $=0$ a 4,9; Insatisfatório $=5,0$ a 7,9; Satisfatório $=8,0$ a 10,0. * $($ manejo de casos x 6$)+($ estrutura $\times 4) \div 10$

\section{P e rcepção dosprofissionais}

\section{Município 1}

\section{PSF comAIDPI}

Os profissionais consideraram o suporte técnico razoável e perceberam a resolubilidade do serviço como boa, mesmo com a falta da contra-referência e a carência de especialistas para referência dos pacientes. Com relação aos aspectos pessoais e interpessoais, o profissional médico encontrava-se satisfeito com a remuneração, porém o profissional de enfermagem mostrou-se insatisfeito pela desigualdade existente para o nível superior. Relataram ótima motivação por ter condições de realizar um bom trabalho e consideraram excelente o relacionamento com pacientes e colegas:

"... como já trabalhamos há seis anos com a mesma comunidade, o relacionamento é exce lente".

\section{PSF semAIDPI}

Os profissionais consideraram o suporte técnico adequado. Com relação à resolubilidade do serviço consideraram-na boa embora com carência de vagas para a referência dos casos. Para os aspectos pessoais e interpessoais, encontravam-se satisfeitos com a remuneração. Tinham boa motivação para o trabalho e bom relacionamento com pacientes e colegas:
"... deve sempre haver motivação com os funcionários e criatividade para estimulá-los";

"... muito agradável e com boa comunicação".

\section{Unidade tradicional}

Dois dos cinco profissionais que participaram do estudo emitiram opinião. Um primeiro referiu considerar o suporte técnico insuficiente quanto a recursos humanos, material de apoio e espaço físico. A resolubilidade do serviço é pouco efetiva, pois os diversos profissionais atuam de forma independente. Quanto aos aspectos pessoais e interpessoais, o profissional encontrava-se satisfeito com a remuneração, porém expressou não estar suficientemente motivado para o trabalho em decorrência da falta de integração da equipe e da ausência de programas por parte da secretaria municipal de saúde que considerem uma melhor abordagem dos problemas da comunidade. Para um segundo profissional, a resolubilidade era satisfatória e ele mesmo considerava-se motivado na profissão. Expressaram, ambos, um bom relacionamento com pacientes e colegas.

\section{Município 2}

\section{PSF com AIDPI}

Apenas o profissional médico participou. Considerou a unidade de saúde com uma boa resolubilidade, recebendo um bom apoio da secretaria municipal de 
saúde. Tem boa motivação para o trabalho que realiza e bom relacionamento com colegas e usuários.

\section{PSF sem AIDPI}

Apenas o profissional de enfermagem emitiu opinião. Quanto ao suporte técnico, considerou como um entrave a dificuldade na contra-referência. Relatou ser a resolubilidade do serviço razoável, traduzindo-se na dificuldade de consultas em conseqüência do número elevado de famílias:

"... são quase 1500 famílias, acima do preconizado pelo Ministério, tentamos fazer o melhor, na medida do possivel".

Para os aspectos pessoais e interpessoais referiu uma demanda excessiva de trabalho burocrático para a enfermagem e, em conseqüência disso, uma menor dedicação para outras atividades como a visita domiciliar. Referiu que a remuneração poderia ser melhor, especificamente para o auxiliar de enfermagem e os agentes comunitários. Sentia-se motivado para o trabalho, mencionou bom relacionamento com colegas e comunidade, além de receber um bom apoio da secretaria municipal de saúde.

\section{Unidade tradicional}

O profissional informou ser o suporte técnico inexistente, dependendo exclusivamente dos profissionais. Considerou o serviço com uma boa resolubilidade e, quanto aos aspectos pessoais e interpessoais referiu remuneração adequada, boa motivação pessoal e bom relacionamento com usuários e colegas.

\section{P e rcepção dos usuários}

Foram realizadas 29 e 24 reuniões em grupos focais nas unidades de saúde respectivamente para os Municípios 1 e 2.

\section{Município 1}

\section{Sobre oacesso e a resolubilidade}

PSF com AIDPI

Os grupos consideraram bons o acesso e a resolubilidade do serviço, não havendo necessidade de procurar outro serviço: "... atende mesmo sem marcar quando tem necessidade". Foi bem enfatizada a visita domiciliar da equipe e não apenas do agente comunitário de saúde (ACS):

"... se a gente está doente, a doutora vai na casa, se precisar uma injeção eles aplicam."
Entretanto, em sete dos 11 grupos, os participantes expressaram que, muitas vezes, há dificuldades de acesso e resolução dos problemas em decorrência da não existência de substituição quando os profissionais se encontram ausentes:

".. é só uma médica pra tudo, deveria ter substi tuto quando fosse fazer um curso, tivesse de férias, pra gente não ficar sem atendimento".

Especialmente com relação ao profissional médico:

"... a médica não está, encaminha pra poli-

clínica. Lá o médico diz que esse problema não é pra ele, é pro posto".

Em um dos grupos houve referência à necessidade de disponibilizar atendimento pediátrico todos os dias: "... as crianças sempre adoecem mais".

Foram referidos, como aspectos negativos, o não funcionamento do serviço em dias feriados e finais de semana e a atenção odontológica: dificuldades para vagas como conseqüência de ser a odontóloga responsável também por uma outra área de abrangência:

"...o defeito aqui é somente o dentista";

"... deveria se dar prioridade pras pessoas que são da área e colocar outro dentista no outro $P S F^{\prime \prime}$;

"... esse programa do dentista devia ser melhor organizado, quando a gente precisa tem que ir pra outro lugar".

PSF sem AIDPI

Para a maioria dos participantes, o acesso e a resolubilidade da unidade mostravam-se precários. Foi enfatizado o pequeno vínculo da comunidade com o serviço de saúde e dificuldades de agendamento, especialmente com relação à assistência à criança:

"... só atende a criança se em caso de emergência"

"... seria muito necessário um pediatra, pois aqui tem muita criança"

".. sentimos falta de alguém que acompanhe a criança, a gente ficava mais seguro"

"... a médica é muito boa, atende bem, examina bem, mas não gosta de atender crianças, não se sente bem"

"... se o filho da gente tiver um problema mais sério, a gente procura logo outro lugar pra atender"

"... pra agendar uma consulta demora e o agente de saúde não passa todo mês na casa da gente";

"... um médico pra tudo é ruim".

Os participantes referiram ainda como aspectos negativos as mudanças freqüentes do profissional 
médico, o não funcionamento do serviço em dias feriados e finais de semana e a ausência de atenção odontológica. Embora apresentando problemas de acesso e resolubilidade, em todos os grupos os participantes informaram utilizar a unidade de saúde para vacinação, referindo atendimento muito bom por parte da auxiliar de enfermagem.

\section{Unidade tradicional}

Os grupos consideraram a resolubilidade boa:

"... a pediatria aqui é muito bom, sempre resolve os problemas"; "o atendimento pra criança é bom";

"... as medicações mais comuns a gente tem aqui e também os exames".

Entretanto, referiram problemas no acesso decorrentes de dificuldades para o agendamento de consultas e o não funcionamento do serviço 24 horas:

"...ruim é pra pegar ficha, tem que chegar cedo, de madrugada e precisa mais médicos, é muita criança".

\section{So b re os modelos de atenção à saúde}

\section{PSF comAIDPI}

Para a maioria dos grupos, os participantes foram capazes de identificar o significado do PSF, alguns referindo como "posto de saúde da família", outros como "programa de saúde da família". Considerando as características de uma unidade do PSF e aquelas de um serviço tradicional, todos os grupos souberam apontar algumas diferenças, focalizadas especificamente no acesso (forma de agendamento de consultas, cadastro), na existência da visita domiciliar e na relação interpessoal:

"... no PSF o atendimento é mais fácil, cadastra a família toda, não precisa madrugar"

"... aqui no PSF tem mais chance, é como uma família, os profissionais vão atrás e se interessam por nós"

"... no PSF tratam melhor a gente, conversam mais e tem as visitas da médica e da enfermeira na casa da gente, nunca se viu isso antes!"

"... também se precisar consulta com especial ista, no PSF já deixa marcada".

PSF sem AIDPI

Em todos os grupos, os participantes não souberam definir PSF, porém expressaram algumas percepções sobre diferenças entre uma unidade pertencente ao
PSF e uma tradicional, centradas, essencialmente, na presença da visita domiciliar e melhor acesso (agendamento de consultas) ao serviço com PSF.

\section{So b re a satisfação do usuário}

\section{PSF com AIDPI}

Os usuários avaliaram o atendimento e o relacionamento com a equipe de forma muito positiva:

"... o atendimento da médica e da enfermeira é excelente!"

"... se interessam e escuta o que a gente fala, responde as nossas perguntas"

"... as meninas (ACS) atende muito bem, a médica trata a gente muito bem".

\section{PSF semAIDPI}

Embora apresentando uma vinculação deficiente com o serviço, pôde-se observar em todos os grupos uma boa relação interpessoal entre os usuários e os profissionais, em que pese o reconhecimento dos usuários quanto às dificuldades na atenção à criança:

"... a médica atende muito bem, examina $e$ explica tudo pra gente"

"... a médica é uma pessoa muito boa".

Também avaliaram como bom, o atendimento no serviço.

\section{Unidade tradicional}

$\mathrm{O}$ atendimento e o relacionamento interpessoal entre usuários e profissionais foram avaliados como bons, particularmente os pediatras e odontólogos:

"... gosto muito dos pediatras daqui, mais do que os particulares"

"... tem um pediatra que gosto muito (várias citações), ele examina a criança, olha o ouvido" "... a enfermeira tem muita paciência com a gente"

"... os dentistas também são muito bons"

"... o pessoal que fica na recepção faz o que pode, às vezes não depende só deles".

Por outro lado, em três grupos houve referências negativas quanto ao relacionamento dos funcionários da recepção e de alguns profissionais médicos com o público:

"... o atendimento de alguns médicos é bom, mas não falam direito com a gente, a gente tem o di reito de saber sobre o problema de saúde"

"... pra mim tem que ter atenção, tratar bem e, às vezes, as atendentes não tratam bem". 
Foi observada também uma carência de diálogo com o público:

"... não é um problema só da obrigação, mas precisa um tratamento mais humano".

\section{Município 2}

\section{Sobreoacesso e a resolubilidade}

PSF comAIDPI

Os participantes consideraram como adequados o acesso e a resolubilidade do serviço:

"... esse posto é bom porque é perto da casa da gente, a gente não precisa andar muito pra se consultar".

Mencionaram, também, a facilidade para agendar consultas e serem atendidos:

"... aqui é ótimo, chega e pega a ficha, ficou muito bom pra gente aqui".

Referiram ser o primeiro serviço que procuram, exceto nos horários sem funcionamento ou ausência dos profissionais:

"... de noite não tem médico aqui, aí a gente procura o hospital, a maternidade"

"... amanhã mesmo o médico não tá aqui, na sexta ele não vem, por isso que eu vim hoje pra pegar o remédio da pressão"

Perceberam o serviço com boa resolubilidade:

"... gosto muito daqui, a gente não precisa ir para a maternidade nem para o hospital, resolve tudo aqui e quando precisa de alguma coisa que aqui não tem, o médico encaminha"

"... tem as visitas dos agentes de saúde, do médico e da enfermeira na casa da gente, que é muito bom".

Porém declararam que o não funcionamento do mesmo no período noturno e finais de semana e ausência de atenção odontológica reduziam o acesso e a resolubilidade do serviço.

\section{PSF sem AIDPI}

Os usuários consideraram a resolubilidade do serviço boa:

"... consigo resolver meus problemas de saúde aqui nesse posto"

"... acompanho e dou as vacinas aqui"

"... a médica examina a criança muito bem, explica tudo"

"... aqui resolvemos nossos problemas e se precisar, eles encaminham para outro lugar".

Embora referindo facilidade geográfica no acesso, na maioria dos grupos foram enfatizadas dificuldades para o atendimento médico decorrente da demanda de pacientes: "Esse posto é bom porque é perto de casa"

"... o problema é que pra pegar ficha, lugar para o atendimento tem que chegar de madrugada"

"... acho que isso acontece porque é muita gente para atender e a médica atende tudo, criança e adulto"

"... devia ter mais médico, principalmente pedi atra porque tem muita criança nessa área"

"... como o atendimento da médica é bom, as pessoas procuram o posto e por causa disso tem muita gente"

Os usuários revelaram dificuldades para realização de exames complementares e ausência de atenção odontológica.

\section{Unidade tradicional}

Em todos os grupos a resolubilidade do serviço e o acesso foram considerados como bons. Informaram receber com regularidade a visita dos agentes comunitários de saúde. Entretanto, a maioria não realizava acompanhamento regular, mesmo tendo conhecimento sobre a existência de unidades do PSF próximo às residências, como também sobre o ambulatório de pediatria dessa unidade:

"... sempre que venho aqui resolvo o problema, o médico passa o remédio e fica bom"

"... aqui é bom porque é perto de casa. Tem um PSF lá perto, mas prefiro o atendimento daqui, lá eu levo pra vacinar"

"... é melhor vir pra cá do que ir pro hospital, lá espera muito"

"... o atendimento aqui agora tá muito melhor"

"... aqui é perto de casa e é fácil conseguir consulta".

\section{S o b re os modelos de atenção à saúde:}

\section{PSF comAIDPI}

De forma unânime, os participantes não foram capazes de reconhecer o significado do PSF. Um dos grupos observados não reconheceu mesmo a própria unidade como PSF:

"... não sabia que esse posto é PSF"

"... a gente vê a propaganda na televisão, mas nem presta atenção, nem percebe"

Entretanto, alguns percebiam diferenças entre uma unidade pertencente ao PSF e uma tradicional relacionadas ao processo de trabalho dos profissionais e à dinâmica do serviço: 
"... Num posto comum é cada um com seu médico, aqui não, o médico é pra todo mundo"

"... vão na casa da gente, faz curativo, dá injeção, consulta"

"... vão na casa da gente e se não tá, deixa recado pra gente procurar o posto".

\section{PSF semAIDPI}

Apenas em um grupo os participantes conheciam o significado de PSF. Entretanto não foram capazes de identificar diferenças entre uma unidade pertencente ao PSF e uma tradicional. Em um outro grupo consideraram como características para um PSF:

"... o posto tem um número determinado de pessoas pra atender"

"... fica perto das residencias".

\section{So b rea satisfação do usuário}

PSF comAIDPI

Os usuários consideraram o atendimento dos profissionais como muito bom, observando-se uma boa relação interpessoal:

"... aqui todos atendem muito bem, o médico, a enfermeira e as meninas (ACS)"

"... são atenciosos com a gente, atende bem mesmo a gente"

"... o médico é uma boa pessoa"

"... os agentes comunitários são muito bons com as crianças, pesa e acompanha bem elas".

\section{PSF sem AIDPI}

Foi unânime a avaliação positiva sobre o atendimento, o que indica uma boa relação interpessoal, mesmo considerando-se a dificuldade apontada quanto ao acesso:

"... gosto muito do atendimento daqui"

"... a enfermeira e a médica atendem muito bem"

"... eles também fazem visita na casa da gente e é bom"

"... todo mundo nesse posto é muito educado"

"... antes de ter esse posto, tudo era mais dificil, tinha que andar muito".

\section{Unidade tradicional}

Os usuários consideraram-se satisfeitos com o atendimento:

"... o atendimento aqui é bom"

"... gosto muito desse posto, tudo organizado e limpo"
"... se precisar internar aqui tem, tem os remé dios e faz os exames também".

Embora para a maioria dos participantes os profissionais atendam bem, alguns depoimentos enfatizaram problemas nas relações interpessoais:

"... muitas vezes depende do médico, como ele se relaciona com a gente"

"... tem médico que nem olha pra gente, não explica direito"

"... o atendimento no hospital é melhor do que aqui, mas lá tem que esperar muito"

"... as pessoas do laboratório não atende bem, não trata bem a gente".

\section{Discussão}

Definir o grau de implantação ou de implementação de uma intervenção é etapa prioritária para a análise do processo, pois

"... não se pode assegurar a eficácia das inter venções se não se souber antes quais são suas características de implantação, ou seja, o grau de implementação e os fatores que favorecem sua dinâmica interna"(Hartz et al.; 1997: 96). 10

Nesse estudo foram detectados diferentes graus de implantação, considerando-se os diferentes meios de implantação com características semelhantes. É evidente o papel positivo do Programa Saúde da Família, especificamente para o Município 1, onde o grau de implantação se apresentou insatisfatório apenas na unidade com o modelo tradicional, a despeito de serem os profissionais médicos pediatras que realizavam a atenção à criança nessa unidade. Ao se observar os resultados de forma imbricada, considerando o grau de implantação e a percepção dos profissionais e usuários, observa-se uma concordância maior entre os achados para as unidades tradicionais nos dois casos avaliados.

O PSF, especialmente pelos mecanismos de adscrição territorial e unidade familiar, tem a potencialidade de contribuir para que as ações de saúde se apresentem mais efetivas. Há um maior contato e atuação mais próxima junto às pessoas, proporcionando um trabalho mais equânime no sentido de inclusão daqueles em situações mais precárias e mais vulneráveis. ${ }^{11}$

É importante referir a influência da atenção proporcionada pelos profissionais de enfermagem seguindo a sistematização da estratégia AIDPI nas unidades do PSF e da unidade tradicional do Município 1. Para o Município 2, é possível que, como os profissionais de enfermagem não realizavam atendimento ao grupo infantil, tal comportamento também tenha influenciado o grau de implantação 
insatisfatório observado para as três unidades. Como os profissionais médicos não realizavam a atenção à criança seguindo a sistematização da estratégia AIDPI, não foi possível definir a real influência dessa estratégia no grau de implantação encontrado. Autores têm encontrado associação positiva da utilização da estratégia AIDPI e o desempenho profissional. 12

Entretanto, intervenções únicas têm-se mostrado incapazes de promover mudanças no comportamento profissional. 13 Os profissionais utilizam diversas abordagens, muitas delas consolidadas mais em crenças do que em evidências científicas. É necessário maior clareza na implantação no contexto da prática profissional assim como o desenvolvimento de intervenções a exemplo de treinamento, supervisão, suporte profissional e incentivos. ${ }^{14,15}$

Embora o instrumento de coleta de dados utilizado para avaliar o manejo de casos tenha sido adaptado do instrumento utilizado para o seguimento da estratégia AIDPI, poderia se discutir o fato de sua aplicação para aqueles profissionais não treinados na AIDPI ou que não utilizam a estratégia, induzir a um erro de avaliação. Tal fato, também observado por Rowe et al.,15 é pouco provável, já que a estratégia AIDPI integra o manejo e condutas de problemas prevalentes na infância largamente conhecidos e utilizados há um longo tempo. Lambrechts et al. 16 comentam que as recomendações para o manejo de casos das doenças diarréicas e das infecções respiratórias agudas para a estratégia AIDPI são semelhantes àquelas já adotadas previamente pela Organização Mundial de Saúde.

Quanto à estrutura dos serviços, em ambos os municípios os escores apresentaram níveis adequados (exceto em uma unidade, mas bem próximo ao nível satisfatório). As variações aconteceram essencialmente em decorrência de problemas relacionados à organização dos serviços, especificamente dificuldades com o sistema de referência e contra-referência mencionadas pelos profissionais e usuários das unidades de saúde. Para Donabedian, 17 as relações entre os aspectos que conformam a estrutura e o processo de atenção à saúde não são tão evidentes. O autor considera as características estruturais como um instrumento 'embotado', inflexível para a avaliação da qualidade, porém imprescindível para o sistema de saúde.

Estudos têm demonstrado avaliações da estrutura dos serviços em níveis adequados de implantação.7,15,16 No entanto, Lambrechts et al. 16 chamam a atenção para o fato de que, além da necessidade de recursos adequados, um dos desafios a serem enfrentados seria o adequado suporte por parte do sistema de saúde, incluído aqui o sistema de referência e contra-referência.

Considerando a política da atenção básica à saúde no Brasil, onde, respeitando-se um dos princípios do SUS, o da integralidade, é preciso também uma integralidade entre os demais âmbitos de atenção. Marques e Mendes 18 sinalizam o empenho do Ministério da Saúde nos últimos anos no que se refere à atenção básica com a expansão do PSF, porém sem garantias de um melhor acesso da população aos âmbitos de maior complexidade de atenção à saúde.

As habilidades de comunicação e as relações interpessoais foram incluídas no estudo para a avaliação do grau de implantação, considerando apenas as variáveis "orientações sobre o tratamento", "perguntas de verificação" e a "compreensão dos usuários após o atendimento". Os resultados evidenciam uma atuação muito mais curativa observada na quase total ausência do recurso a perguntas de verificação que poderiam ser um veículo a mais para uma melhor interação com os usuários. Esse fato também pode ser observado pelos escores adequados quanto aos procedimentos terapêuticos e pelos escores baixos para as ações de prevenção de doenças e promoção da saúde. Essa constatação é refletida ao se observar que também os usuários apreendem mais facilmente os aspectos curativos da atenção à saúde.

A segunda dimensão incorporada ao primeiro nível de análise, isto é, a percepção dos profissionais e usuários, surge como um resultado da atenção à saúde e um importante componente explicativo e imbricado na avaliação do grau de implantação.

Constata-se um predomínio de fatores ligados à organização dos serviços como causas para as dificuldades no acesso aos cuidados de saúde. Para os usuários, a grande demanda, o não funcionamento das unidades nas 24 horas e durante toda a semana, além de aspectos relacionados diretamente aos profissionais, como ausências e dificuldades no atendimento ao grupo infantil, são os fatores que dificultam um melhor acesso e resolubilidade. É interessante referir a percepção desses usuários quanto ao forte papel atribuído ao grupo infantil por uma maior demanda por cuidados de saúde. Um outro ponto relacionado ao acesso encontra-se na ênfase à visita domiciliar como um aspecto positivo. Também os profissionais percebem a grande demanda dos serviços e as dificuldades com o sistema de referência e contra-referência como fatores negativos à qualidade e à resolubilidade da atenção prestada. Estudos têm constatado inadequações na relação entre equipe e população e na dimensão organizacional, sendo esses aspectos percebidos como prejudiciais ao acesso e à integrali- 
dade das ações. Também verificaram ser a visita domiciliar a atividade mais positivamente enfatizada. 19,20

Sobre os modelos de atenção à saúde, foi percebido um melhor reconhecimento quanto ao significado e características do PSF para a unidade PSF com AIDPI do Município 1, onde não havia mudanças recentes da equipe de saúde e tanto o médico como o enfermeiro prestavam atendimento regular à saúde da criança. Dificuldade semelhante foi observada por Trad et al. 20

Mesmo reconhecendo a importância da dimensão interpessoal para o adequado cuidado à saúde, há uma certa dificuldade em incluí-la nos estudos de avaliação. Donabedian 17 observa que as informações não são facilmente quantificáveis, os critérios e padrões são variáveis e não bem desenvolvidos. Por ser o processo interpessoal extremamente flexível e influenciado por diversos fatores intrínsecos às pessoas e ao contexto, ele deve ser adaptado às variações de expectativas que acontecem tanto para os usuários como para os profissionais.

Observa-se uma estreita associação entre o tipo de serviço e a vinculação das pessoas ao mesmo. Para ambos os casos aqui estudados há uma evidente associação entre uma melhor relação interpessoal e o fato de ser unidade do PSF, a despeito de uma das unidades (PSF sem AIDPI do Município
1) ter apresentado maior fragilidade no vínculo da população com o serviço. Identifica-se também uma percepção semelhante por parte dos profissionais quando os mesmos refletem sobre a fragmentação e o pouco suporte técnico dos processos de trabalho nas unidades tradicionais.

Os usuários tendem a considerar as atitudes de compreensão, de acolhimento e de comunicação dos profissionais, além do desempenho clínico no sentido de que o problema de saúde seja solucionado. Há dois elementos-chave: a efetividade clínica e a efetividade das relações interpessoais. Aspectos como humanismo, sensibilidade e receptividade são essenciais aos profissionais para uma boa relação interpessoal.21-23

O estudo possibilitou observar que, para os dois municípios considerados em um nível de análise centrado nos serviços de saúde, a variação no grau de implantação apresentou como fatores determinantes, deficiências no manejo de casos e a potencialidade da atenção prestada pelos profissionais de enfermagem. Porém não foi possível definir a influência da estratégia AIDPI. Também se observou melhor satisfação profissional e dos usuários para unidades do PSF, entretanto com deficiências no acesso e na integralidade entre a atenção primária e demais níveis de atenção independente do tipo de serviço de saúde para ambos os casos.

\section{Referências}

1. Ministério da Saúde, Instituto Materno Infantil de Pernambuco (IMIP), Universidade Federal de Pernambuco, Departamento de Nutrição, Secretaria Estadual de Saúde de Pernambuco. Atenção à Saúde Materno-Infantil no Estado de Pernambuco. Recife: Bagaço; 2000.

2. Casanova C, Starfield B. Hospitalizations of children and access to primary care: a cross-national comparison. Int J Health Serv 1995; 25: 283-94.

3. Ministério da Saúde. Secretaria de Políticas de Saúde. Área de Saúde da Criança. O Brasil e a Atenção Integrada às Doenças Prevalentes da Infância. Brasília (DF); 1997.

4. Ministério da Saúde. Secretaria de Assistência à Saúde. Coordenação de Saúde da Comunidade. Saúde da família: uma estratégia para a reorientação do modelo assistencial. Brasília (DF); 1997.

5. Ministério da Saúde. Secretaria de Assistência à Saúde. Coordenação de Atenção Básica. Avaliação da Implantação e Funcionamento do Programa de Saúde da Família (PSF). Brasília (DF); 2000.
6. Bodstein R. Atenção básica na agenda da saúde. Cienc Saúde Coletiva 2002; 7: 401- 2

7. Felisberto E, Carvalho EF, Maggi RS, Samico I. Avaliação do processo de implantação da Estratégia da Atenção Integrada às Doenças Prevalentes da Infância no Programa Saúde da Família no estado de Pernambuco. Cad Saúde Pública 2002; 18: 1737- 45.

8. Ministério da Saúde. Secretaria de Políticas de Saúde. Área de Saúde da Criança, OPAS (Organização Panamericana da Saúde). Manual de acompanhamento e avaliação da Atenção Integrada às Doenças Prevalentes da Infância (AIDPI). Brasília (DF); 1999.

9. Krueger RA. Focus groups: a practical guide for applied research. 2. ed. London: SAGE; 1994.

10. Hartz ZMA, Champagne F, Contandriopoulos AP, Leal MC. Avaliação do Programa Materno-Infantil: análise de implantação em sistemas locais de saúde no Nordeste do Brasil. In: Hartz ZMA, organizadora. Avaliação em saúde: dos modelos conce ituais à prática na análise da implantação 
de programas. Rio de Janeiro: FIOCRUZ; 1997. p. 89-131.

11. Senna MCM. Eqüidade e política de saúde: algumas reflexões sobre o Programa Saúde da Família. Cad Saúde Pública 2002; 18 ( Supl): 203-11.

12. Amaral J, Gouws E, Bryce J, Leite AJM, Cunha ALA, Victora CG. Effect of Integrated Management of Childhood Illness (IMCI) on health worker performance in NortheastBrazil. Cad Saúde Pública 2004; 20 ( Supl 2): S209-S19.

13. Jones R. Primary care research: ends and means. Fam Pract 2000; 17: 1-4.

14. Grol R. Beliefs and evidence in changing clinical practice. Br Med J 1997; 315: 418- 21.

15. Rowe AK, Onikpo F, Lama M, Cokou F, Deming MS Management of childhood illness at health facilities in benin: problems and their causes. Am J Public Health 2001; 91: $1625-35$.

16. Lambrechts T, Bryce J, Orinda V. Integrated management of childhood illness: a summary of first experiences. Bull World Health Organ 1999; 77: 582-94.

17. Donabedian A. The quality of care - How can it be assessed? JAMA 1988; 260: 1743-8.
18. Marques RM, Mendes A. A política de incentivos do Ministério da Saúde para a atenção básica: uma ameaça à autonomia dos gestores municipais e ao princípio da integralidade? Cad Saúde Pública 2002; 18 ( Supl): 163-71.

19. Conill EM. Políticas de atenção primária e reformas sanitárias: discutindo a avaliação a partir da análise do Programa Saúde da Família em Florianópolis, Santa Catarina, Brasil, 1994 - 2000. Cad Saúde Pública 2002; 18 (Supl): 191-202.

20. Trad LAB, Bastos ACS, Santana EM, Nunes MO. Estudo etnográfico da satisfação do usuário do Programa de Saúde da Família (PSF) na Bahia. Cienc Saúde Coletiva 2002; 7: 581-9.

21. Campbell SM, Roland MO, Buetow SA. Defining quality of care. Soc Sci Med 2000; 51: 1611-25.

22. Campbell SM, Braspenning J, Hutchinson A, Marshall M. Research methods used in developing and applying quality indicators in primary care. Qual Saf Health Care 2002; 11: 358-64.

23. Ramos DD, Lima MADS. Acesso e acolhimento aos usuários em uma unidade de saúde de Porto Alegre, Rio Grande do Sul, Brasil. Cad Saúde Pública 2003; 19: 27- 34.

Recebido em 14 de fevereiro de 2005

Versão final apresentada em 4 de abril de 2005

Aprovado em 25 de abril de 2005 